\title{
PULSE COMPRESSION IN A PASSIVELY MODE-LOCKED RUBY LASER
}

\author{
P. SPERBER and A. PENZKOFER \\ Naturwissenschaftliche Fakultät II - Physik, Universität Regensburg, 8400 Regensburg, Fed. Rep. Germany
}

Received 11 March 1985

\begin{abstract}
A passively mode-locked ruby laser oscillator with two saturable absorber cells is described that towards the end of the pulse train, generates picosecond pulses of about 3 ps FWHM upon weak pedestals approximately 40 ps wide. The pulse shortening in the oscillator is a combined effect of pulse chirping by self-phase modulation, pulse compression of the chirped pulses by the finite spectral gain width of the active medium, and pulse shortening by the saturable absorbers.
\end{abstract}

\section{Introduction}

In passively mode-locked ruby lasers (saturable dye DDI in methanol) pulse duration between 40 ps and 2 ps have been reported (around 30 ps in refs. [1-4], below $10 \mathrm{ps}$ in refs. [5-8]). Here we analyse the temporal pulse development along the pulse train for an oscillator with a single saturable dye cell (conventional system) and two saturable dye cells (higher total dye concentration). While in the conventional system the pulse durations are about 30 ps along the pulse train, they shorten to about 3 ps toward the end of the train in the oscillator with two dye cells. The short pulses are upon low-intensity pedestals of about 40 ps duration. This broad pulse pedestal is strongly reduced by application of an external saturble absorber. The pulse shortening will be shown to be mainly due to pulse compression of chirped pulses in the active medium of finite spectral gain width [9, $10]$. The wide variation in the pulse duration reported in previous work [1-8] may be understood by the mechanisms described here.

\section{Experimental}

The ruby laser oscillator is sketched in fig. 1 . The resonator is formed by the plane $100 \%$ mirror M1 and the spherical output mirror M2 $(\rho=3 \mathrm{~m})$ of $70 \%$ reflectivity. The mirror spacing is $2.15 \mathrm{~m}$. The active

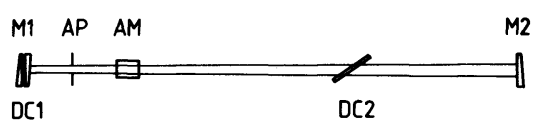

Fig. 1. Picosecond ruby laser oscillator. M1, M2, mirrors; DC1, DC2, dye cells; AM, ruby rod; AP, circular aperture.

medium AM (4 inch length, 1/4 inch diameter, 0.05 weight $\% \mathrm{Cr}_{2} \mathrm{O}_{3}$ ) is located in $40 \mathrm{~cm}$ distance from the front mirror M1. The width of aperture AP is 1.9 $\mathrm{mm}$. The contacted dye cell DC1 contains the modelocking dye DDI (1,1'-diethyl-2,2'-dicarbocyanine iodide) dissolved in methanol (double pass small signal transmission $T_{01}=0.64$ ). For the experiments with two dyes the cell DC2 is positioned under Brewster's angle at a distance of $1.4 \mathrm{~m}$ from the front mirror. DDI dissolved in methanol is used in DC2 (single pass small signal transmission $T_{02}=0.77$ ).

Without taking into account the pulse amplification in the active medium, the pulse intensity is a factor of 4.5 higher in the contacted dye cell than in the laser rod (a factor of 3 results from back reflection [11]), and in dye cell DC2 the intensity is a factor of 2 lower than in the ruby rod (factor of 1.5 comes from Brewster angle). The different intensities in the two dye cells extend the region of optimum pulse shortening in the oscillator.

Single picosecond light pulses at various positions along the pulse train are selected with a krytron triggered Pockels cell shutter. The pulse durations are 
measured by the two-photon fluorescence method [12] $\left(2.5 \times 10^{-3}\right.$ molar rhodamine $6 \mathrm{G}$ dissolved in ethanol is used).

\section{Results}

The performance of the ruby laser without dye cell DC2 (fig. 2) is tested first. A typical pulse train is shown by the vertical lines in fig. $2 a$. The obtained pulse durations along the pulse train are shown by the open circles in fig. $2 b$. They are calculated from the halfwidths $\Delta \tau$ of the two-photon fluorescence signal $S(\tau)$ above constant level by the relation $\Delta t=\Delta \tau / \sqrt{2}$

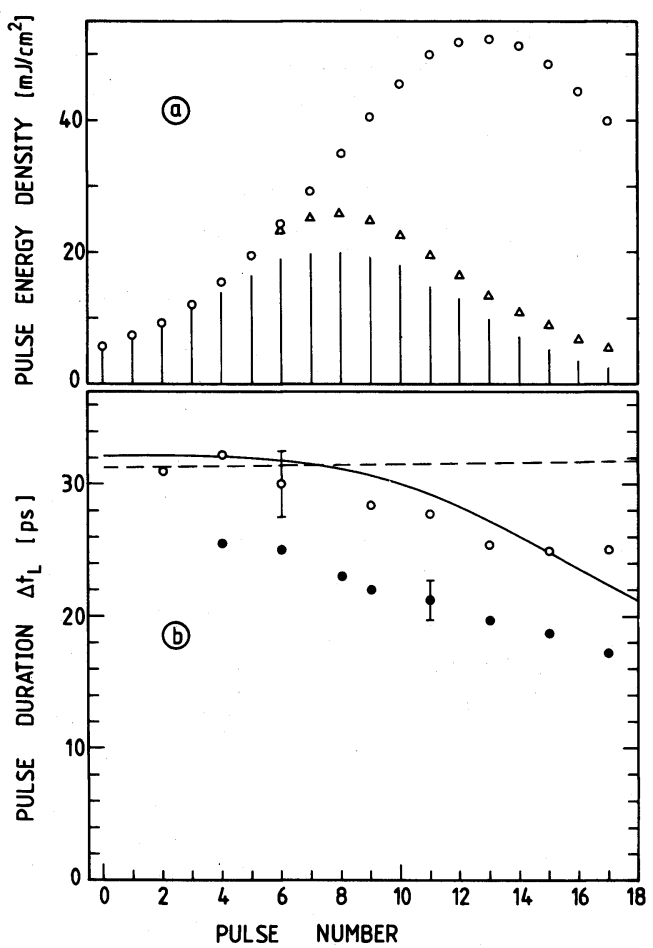

Fig. 2. Pulse development with single dye cell. (a) Peak energy density of pulses along train. Solid lines: experimental train. Circles: calculated train neglecting self-phase modulation. Triangles: calculated train including self-phase modulation with gain adjustment in rising part of train. (b) Pulse durations along train. Open circles: without external dye. Closed circles: with external DDI $\left(T_{0}=0.08\right)$. Dashed curve is calculated excluding self-phase modulation. Solid curve includes self-phase modulation and belongs to the experimental train of (a). which is valid for temporally gaussian shaped pulses. The pulse durations reduce from about 32 ps before the pulse train maximum to about 25 ps towards the end of the pulse train. Bandwidth limited pulses are found in the early part of the pulse train $(\Delta \nu \Delta t \simeq 0.5)$. Towards the end of the train spectral structuring due to self-phase modulation is observed $(\Delta \nu \Delta t \simeq 4)$.

The laser performance with additional dye cell DC2 (DDI in methanol, $T_{02}=0.77$ ) is illustrated in figs. 3-5. The shape of a typical pulse train is shown by the vertical lines of fig. 3a. The pulse height increases approximately a factor of 2.5 compared to the resonator without $\mathrm{DC} 2$. The pulse train rises steep-

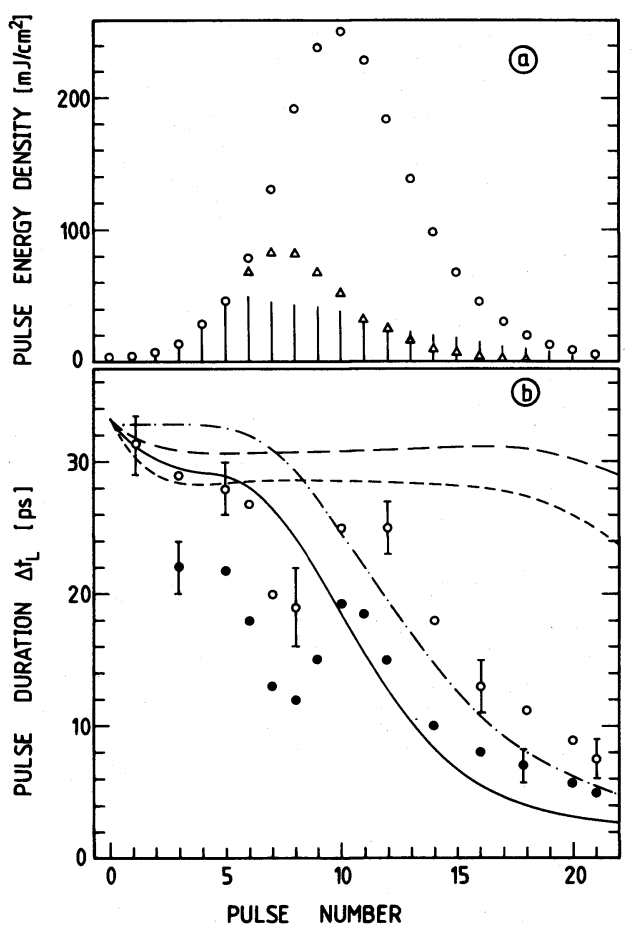

Fig. 3. Pulse development with two dye cells. (a) Pulse trains. Same classification as in fig. 2. (b) Pulse duration along train. Open circles: measured apparent durations without external dye $(\Delta t=\Delta \tau / \sqrt{2})$. Closed circles: measured apparent durations with external DDI dye $\left(T_{0}=0.08\right)$. Curves are calculated. Long-dashed curve: self-phase modulation is neglected [circles of (a)]. Short-dashed curve: without self-phase modulation using the experimetnal pulse intensities of (a). Solid curve, includes self-phase modulation and belongs to the experimental pulse train of (a). Dash-dotted curve, same as solid curve, only pulse shortening by saturable absorbers is excluded. 


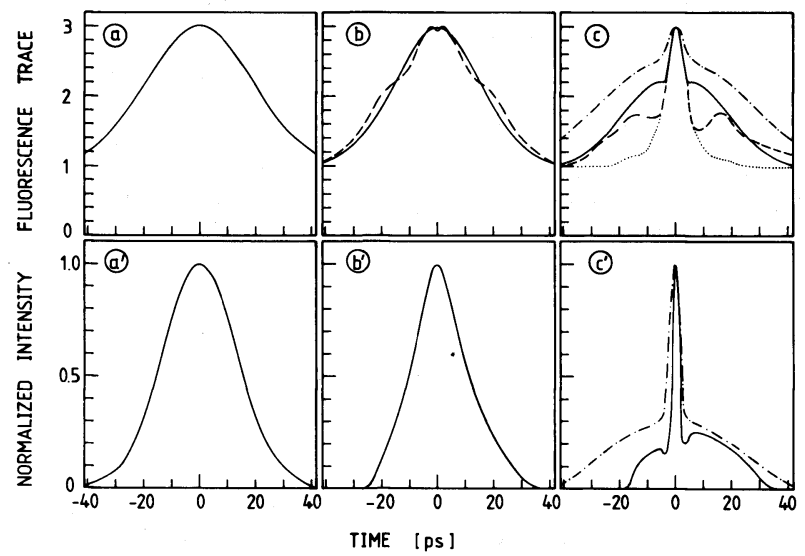

Fig. 4. Two-photon fluorescence traces (a)-(c) and pulse shapes $\left(a^{\prime}\right)-\left(c^{\prime}\right)$. Two dye cells in oscillator. Pictures belong to the experimental train of fig. $3 a$ at positions $0(a),\left(a^{\prime}\right)$, 9 (b), ( $\left.b^{\prime}\right)$ and 21 (c), ( $\left.c^{\prime}\right)$. Dashed curves: experimental traces. Dotted curve: experimental trace after passing through external dye cell $\left(T_{0}=0.08\right)$. Solid curves: calculated including self-phase modulation and saturable absorber action. Dash-dotted curves: calculated in same way as solid curves only neglecting saturable absorber action.

ly, forms a broad maximum and declines slowly. The open circles in fig. $3 \mathrm{~b}$ indicate the measured apparent pulse durations $(\Delta t=\Delta \tau / \sqrt{2})$. In the rising part of the train near the train maximum the pulse durations are around $30 \mathrm{ps}$. They reduce to about $20 \mathrm{ps}$ in the maximum region, then slightly increase before reducing to about 8 ps towards the end of the train.

Two typical two-photon fluorescence traces are shown by the dashed curves in figs. $4 \mathrm{~b}$ and $4 \mathrm{c}$. In the rising part of the train (fig. 4a) the trace has a simple bell shape (coincides with calculated solid curve). Slightly beyond the train maximum the trace forms shoulders (fig. 4b, increased two-photon fluorescence width in fig. $3 \mathrm{~b}$ ). Towards the end of the train the height of the shoulders reduces, the width of the shoulders remains nearly constant, but the central peak sharpens strongly (short pulse of about 3 ps duration up on weak pedestal, see discussion below).

Measured pulse spectra along the pulse train are depicted in figs. $5 \mathrm{a}-$ c. Fig. 5 a shows the nearly bandwidth limited spectrum in the rising part of the pulse train. Fig. $5 \mathrm{~b}$ presents a spectrum at the train maximum. Fig. $5 \mathrm{c}$ gives a strongly structured spectrum towards the end of the pulse train.

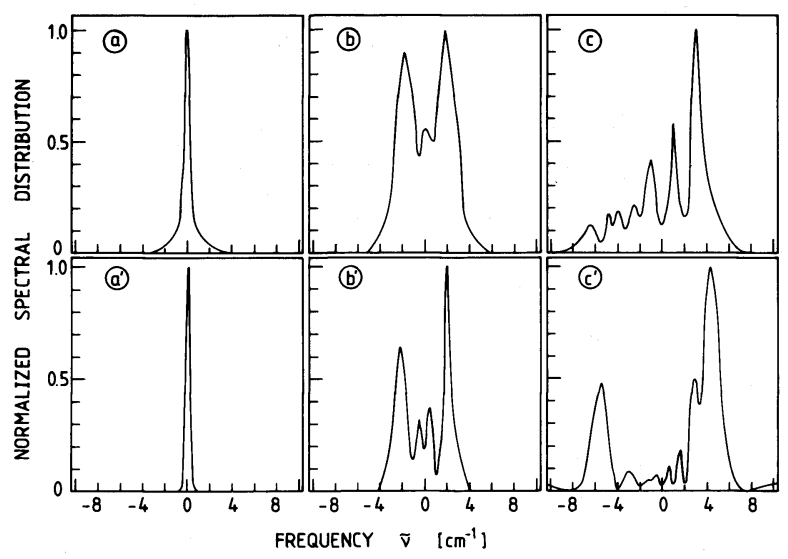

Fig. 5. Pulse spectra with two saturable dye cells. (a)-(c) measured spectra. $\left(a^{\prime}\right)-\left(c^{\prime}\right)$ calculated spectra. They belong to the experimental train of fig. $3 a$ at positions $0(a),\left(a^{\prime}\right)$, 9 (b), (b') and $21(\mathrm{c}),\left(c^{\prime}\right)$.

\section{Discussion}

A detailed theoretical analysis of the picosecond pulse development will be given elsewhere [13]. Here only some theoretical results are shown to clarify the mechanism of short pulse generation in ruby oscillators. The following four situations are discussed:

(i) Pulse development without self-phase modulation and with adjustment of initial population of upper laser level to pulse energy growth in the rising part of the pulse train.

The laser behaviour without the additional saturable absorber DC2 is considered first. The open circles in fig. 2a show the development of pulse energy density $\epsilon(r=0)$ along the pulse train (inside output mirror). The pulse energy increases about a factor of 2.5 above the experimental value.

The dashed curve in fig. $2 \mathrm{~b}$ displays the calculated pulse durations along the pulse train. The curve is fitted to 31 ps at pulse number 0 and remains constant along the train. The peak pulse intensity in the contacted dye cell $\left[I_{0 \mathrm{~L}} \simeq \epsilon(r=0) / \Delta t_{\mathrm{L}} \simeq 2 \times 10^{9} \mathrm{~W} / \mathrm{cm}^{2}\right.$ already for pulse number 0$]$ is far above the saturation intensity of the dye $I_{\mathrm{S}}=h \nu_{\mathrm{L}} / \sigma_{\mathrm{L}} \tau 2.2 \times 10^{7} \mathrm{~W} / \mathrm{cm}^{2}$ (absorption cross section $\sigma_{\mathrm{L}}=7.6 \times 10^{-16} \mathrm{~cm}^{2}$, absorption recovery time $\tau=17 \mathrm{ps}$ [4]) and therefore no pulse shortening by the saturable dye occurs.

With two saturable absorber cells the pulse energies 
along the train would follow the open circles of fig. 3a. The experimental pulse energy density at the train maximum is about a factor of 8 lower than the calculated one. The long-dashed curve in fig. $3 \mathrm{~b}$ indicates that no pulse shortening occurs since the laser intensity in both dye cells is high compared to the saturation intensity.

(ii) Pulse development without self-phase modulation and adjustment of pulse energy densities to experimental values.

When only the contacted dye cell is in the resonator the pulse durations remain unchanged along the pulse train of fig. 2a. In case of two dye cells in the oscillator (short-dashed curve in fig. 3b) a pulse shortening from $33 \mathrm{ps}$ at the beginning of the train to 24 ps towards the end of the train is calculated. The experimentally observed pulse shortening is stronger than the calculated one indicating that the pulse shortening action of the two saturable absorbers alone is not enough to explain the experimental findings.

(iii) Pulse development including self-phase modulation, spectral gain profile and gain adjustment in rising part of pulse train.

The nonlinear refractive index of ruby $\left(\gamma=n_{2} /\right.$ $\left.n_{0} c \epsilon_{0}=3.7 \times 10^{-16} \mathrm{~cm}^{2} / \mathrm{W}, n_{0} \simeq 1.76[14]\right)$ gives rise to self-phase modulation [15] and self-focusing [16]. The self-phase modulation sweeps the carrier frequency of the ligh pulse along time in a sineshaped manner (pulse chirping) [17]. The frequency broadening may extend beyond the spectral width of the gain profile of the active medium (fluorescence linewidth of laser transition of ruby $\Delta \nu_{\mathrm{R}} \simeq 11 \mathrm{~cm}^{-1}$ ) so that the frequency extrema of the chirped pulses observe reduced gain in the oscillator. The corresponding temporal inflection points of the pulses are less amplified and the temporal half-width of the pulses narrow (pulse compression) [10]. The saturable dyes absorb preferentially the temporal wings of the modulated pulses and contribute to the pulse shortening. The calculated curve below are not integrated over the beam profile, but half the $\gamma$-value is used to take into consideration a realistic spatial beam profile.

The triangles in figs. 2a and 3a show the development of the pulse heights along the train. Comparing with open circles [case (i)] indicates that gain reduction due to spectral broadening by self-phase modulation reduces the pulse energies strongly. The small difference compared to the experimental pulse train is thought to be due to self-focusing losses and depolarization losses. The temporal development of the pulse durations and the spectra is similar to case (iv) and is discussed there.

(iv) Pulse development including self-phase modulation, spectral gain profile and pulse energy density adjustment to experimental values.

The solid curve of fig. $2 \mathrm{~b}$ for the pulse durations follows the experimental values rather well (only contacted dye cell). For situation of two saturable absorbers in the oscillator the calculated pulse durations are given by the solid curve of fig. $3 \mathrm{~b}$. The deviation from the experimental points in the plateau region beyond the train maximum is thought to be a little bit due to the additional effect of self-focusing (reduction of peak of circulating pulse) and mainly due to the formation of shoulders in the two-photon fluorescence trace. It should be emphasized that the experimental durations are determined from the halfwidth of the fluorescence trace assuming gaussian temporal pulse shape. This method over-estimates considerably the real pulse width (FWHM) if the pulse is upon a broad pedestal (see below).

Calculated temporal pulse shapes and the corresponding auto-correlation functions are depicted by the solid curves in fig. 4 (pulse numbers 0,9 and 21 of fig. 3a). Slightly beyond the train maximum (figs. $4 b$ and $4 b^{\prime}$ ) the incision of the pulses begins (reduced gain at temporal inflection points). Towards the end of the pulse train (figs. $4 c$ and $4 c^{\prime}$ ) short pulses on weak pedestals are formed.

The calculated two-photon fluorescence traces agree reasonably with the experimental traces. A comparison of the calculated two-photon fluorescence traces

$$
S(\tau)=1+\frac{2 \int_{-\infty}^{\infty} I(t-\tau) I(t) \mathrm{d} t}{\int_{-\infty}^{\infty} I^{2}(t) \mathrm{d} t}
$$

with the corresponding temporal pulse shapes indicates that towards the end of the pulse train short pulses of about 3 ps upon a weak pedestal of about 40 ps duration are generated in the oscillator with two saturable absorber cells.

The temporal wings may be reduced by passing the 
pulses through a saturable absorber outside the oscillator. The full circles in figs. $2 b$ and $3 b$ show the experimentally obtained pulse durations (gaussian shape assumed) along the pulse train when a DDI dye cell of $T_{0}=0.08$ initial transmission was placed behind the Pockels cell single pulse selector. The dotted curve in fig. $4 \mathrm{c}$ shows a measured two-photon fluorescence trace which is obtained towards the end of the pulse train.

The three spectra in figs. $5 \mathrm{a}^{\prime}-\mathrm{c}^{\prime}$ belonging to pulse numbers 0,9 and 21 of fig. 3 a show the spectral pulse development along the pulse train. They indicate strong self-phase modulation at and beyond the pulse train maximum. They are in reasonable agreement with the experimental spectra of figs. $5 \mathrm{a}-\mathrm{c}$.

To separate the influence of the self-phase modulation on pulse shortening from the pulse shortening effect of the saturable dyes calculations were made without including the dye bleaching (experimental pulse energy densities of fig. $3 \mathrm{a}$ are used). The dashdotted curves in figs. $3 \mathrm{~b}, 4 \mathrm{c}, \mathrm{c}^{\prime}$ show that the pulses are only slightly broader and the pedestals are only slightly higher compared to the situation where dye bleaching is included. This result clearly reveals the dominant importance of pulse compression of the chirped pulses by the active medium.

\section{Conclusion}

In resonators with high saturable absorber concentration (e.g. two dye cells in resonator) high peak pulse intensities are generated and strong spectral broadening by self-phase modulation occurs. The compression of the chirped pulses by the finite spectral gain width of the active medium results in efficient pulse shortening in the trailing part of the pulse train. The reported strongly varying pulse durations of different experimental groups [1-8] may be understood by the above described mechanisms.

\section{Acknowledgement}

The authors are grateful to Th. Ascherl for experimental assistance. They thank the Deutsche Forschungsgemeinschaft for financial support and the Rechenzentrum of the University Regensburg for disposal of computer time.

\section{References}

[1] M.W. McGeoch, Optics Comm. 7 (1973) 116.

[2] E.G. Arthurs, D.J. Bradley and T.J. Glynn, Optics Comm. 12 (1974) 136.

[3] H. Tashiro, J. Schulz-Hennig and A. Müller, Optics Comm. 27 (1978) 442.

[4] W. Blau, R. Reber and A. Penzkofer, Optics Comm. 43 (1982) 210.

[5] R. Cubeddu, R. Polloni, C.A. Sacchi and O. Svelto, IEEE J. Quant. Electron. QE-5 (1969) 470.

[6] R. Polloni, IEEE J. Quant. Electron. QE-8 (1972) 428.

[7] I.K. Krasyuk, P.P. Pashkin and A.M. Prokhorov, JETP Lett. 7 (1978) 89.

[8] M.E. Mack, IEEE J. Quant. Electron. QE-4 (1968) 1015.

[9] H. Nathel, D.M. Guthals and J.H.I. Clark, Technical Digest of CLEO'84 (Anaheim, California, June 1984) p. 182.

[10] A. Penzkofer and F. Graf, Opt. Quant. Electron., to be published.

[11] J. Herrmann, F. Weidner and B. Wilhelmi, Appl. Phys. B26 (1981) 197.

[12] J.A. Giordmaine, P.M. Rentzepis, S.L. Shapiro and K.W. Wecht, Appl. Phys. Lett. 11 (1967) 216.

[13] A. Penzkofer and P. Sperber, to be published.

[14] M.J. Moran, C.Y. She and R.L. Carman, IEEE J. Quant. Electron. QE-11 (1975) 259.

[15] F. Shimizu, Phys. Rev. Lett. 19 (1967) 1097.

[16] R.Y. Chiao, E. Garmire and C.H. Townes, Phys. Rev. Lett. 13 (1964) 479.

[17] E.B. Treacy, Phys. Lett. 28A (1968) 34. 